Published in final edited form as:

J Geriatr Oncol. 2015 March ; 6(2): 119-126. doi:10.1016/j.jgo.2014.11.002.

\title{
Prophylactic Cranial Irradiation in Elderly Patients with Small Cell Lung Cancer: Findings from a North Central Cancer Treatment Group Pooled Analysis
}

\author{
W. G. Rule ${ }^{a}$, N. R. Foster ${ }^{b}$, J. P. Meyers ${ }^{b}$, J. B. Ashman ${ }^{a}$, S. A. Vora ${ }^{a}$, T. F. Kozelsky ${ }^{c}$, Y. I. \\ Garces $^{\mathrm{c}}$, J. J. Urbanic ${ }^{\mathrm{d}}$, J. K. Salama ${ }^{\mathrm{e}}$, and S. E. Schild ${ }^{\mathrm{a}}$ \\ aDepartment of Radiation Oncology, Mayo Clinic Arizona \\ bSection of Biomedical Statistics and Informatics, Mayo Clinic Rochester \\ 'Department of Radiation Oncology, Mayo Clinic Rochester \\ dDepartment of Radiation Oncology, Wake Forest School of Medicine \\ eDepartment of Radiation Oncology, Duke University School of Medicine
}

Corresponding Author: Dr. William G. Rule, Mayo Clinic, Department of Radiation Oncology, 5777 East Mayo Blvd., Phoenix, AZ 85054, Telephone: (480) 342-1262, Fax: (480) 342-3972, rule.william@mayo.edu.

Publisher's Disclaimer: This is a PDF file of an unedited manuscript that has been accepted for publication. As a service to our customers we are providing this early version of the manuscript. The manuscript will undergo copyediting, typesetting, and review of the resulting proof before it is published in its final citable form. Please note that during the production process errors may be discovered which could affect the content, and all legal disclaimers that apply to the journal pertain.

Authors \& Disclosures

1. William G. Rule, M.D. - Mayo Clinic, Scottsdale, AZ, USA

a. No disclosures

2. Nathan R. Foster - Mayo Clinic, Rochester, MN, USA

a. No disclosures

3. Jeffrey P. Meyers - Mayo Clinic, Rochester, MN, USA

a. No disclosures

4. Timothy Kozelsky, M.D. - Mayo Clinic, Rochester, MN, USA

a. No disclosures

5. Jonathan Ashman, M.D., Ph.D. - Mayo Clinic, Scottsdale, AZ, USA

a. No disclosures

6. Sujay Vora, M.D. - Mayo Clinic, Scottsdale, AZ, USA

a. No disclosures

7. Yolanda I. Garces, M.D. - Mayo Clinic, Rochester, MN, USA

a. No disclosures

8. James J. Urbanic, M.D. - Wake Forest School of Medicine, Winston Salem, NC, USA

a. No disclosures

9. Joseph K. Salama, M.D. - Duke University School of Medicine, Durham, NC, USA

a. No disclosures

10. Steven E. Schild, M.D. - Mayo Clinic, Scottsdale, AZ, USA

a. No disclosures

Disclosures and Conflict of Interest Statements

The authors have declared no conflicts of interest.

Author Contributions

Study Concept and Design: SE Schild, WG Rule, NR Foster, TF Kozelsky, JP Meyers

Data Acquisition: NR Foster and JP Meyers

Quality Control of Data and Algorithms: NR Foster and JP Meyers

Data Analysis and Interpretation: All authors

Statistical Analysis: NR Foster and JP Meyers

Manuscript Preparation: WG Rule

Manuscript Editing: All authors

Manuscript Review: All authors 


\section{Abstract}

Objectives-To examine the efficacy of prophylactic cranial irradiation (PCI) in elderly patients with small cell lung cancer (SCLC) ( $\geq 70$ years of age) from a pooled analysis of four prospective trials.

Materials \& Methods-One hundred fifty-five patients with SCLC (limited stage, LSCLC, and extensive stage, ESCLC) participated in four phase II or III trials. Ninety-one patients received PCI (30Gy/15 or $25 \mathrm{~Gy} / 10)$ and 64 patients did not receive PCI. Survival was compared in a landmark analysis that included only patients who had stable disease or better in response to primary therapy.

Results-Patients who received PCI had better survival than patients who did not receive PCI (median survival 12.0 months vs. 7.6 months, 3 -year overall survival $13.2 \%$ vs. $3.1 \%, \mathrm{HR}=0.53$ [95\% CI 0.36-0.78], $p=0.001$ ). On multivariate analysis of the entire cohort, the only factor that remained significant for survival was stage (ESCLC vs. LSCLC, $p=0.0072$ ). In contrast, the multivariate analysis of patients who had ESCLC revealed that PCI was the sole factor associated with a survival advantage (HR=0.47 [95\% CI 0.24-0.93], $p=0.03)$. Grade 3 or higher adverse events (AEs) were significantly greater in patients who received PCI (71.4\% vs. $47.5 \%$, $p=0.0031$, with non-neuro and non-heme being the specific AE categories most strongly correlated with PCI delivery.

Conclusions-PCI was associated with a significant improvement in survival for our entire elderly SCLC patient cohort on univariate analysis. Multivariate analysis suggested that the survival advantage remained significant in patients with ESCLC. PCI was also associated with a modest increase in grade 3 or higher AEs.

\section{Keywords}

Prophylactic cranial irradiation; PCI; small cell lung cancer

\section{Introduction}

Prophylactic cranial irradiation (PCI) is standard therapy in the management of patients with limited stage small cell lung cancer (LSCLC) or extensive stage small cell lung cancer (ESCLC) who have experienced any degree of favorable response to initial therapy.(1-7) In spite of the proven survival benefit, many eligible patients with SCLC do not receive PCI due to patient or physician concerns regarding neurotoxicity. In clinical practice, the dilemma of whether to administer this life-prolonging therapy is most controversial in older individuals. Lung cancer is a disease which is prevalent in the elderly, with a median age at diagnosis of approximately 70 years. Unfortunately, most of the trials that have led to the adoption of PCI enrolled relatively few elderly patients. The risk benefit analyses for these patients is particularly challenging as the survival benefit for PCI was seen at 1 year following PCI for LSCLC(2), although much sooner in ESCLC.(4) Previously, we analyzed the effect of PCI in a large cohort of patients with both LSCLC and ESCLC, confirming the benefit in both patient populations.(3) The purpose of this study was to specifically examine the results of PCI in patients with SCLC (both LSCLC and ESCLC) who are elderly ( $\geq 70 \mathrm{y}$ ) in order to evaluate whether the survival benefit from PCI is also present in older patients. 


\section{Materials \& Methods}

The patient population included patients 70 years of age or older with LSCLC or ESCLC who participated in four prospective phase II or III North Central Cancer Treatment Group trials (86-20-51, 89-20-51, 89-20-52, 95-20-53). Patients with stable disease or better following chemotherapy $+/-$ thoracic radiotherapy (TRT) were included. Response assessment was based on history and physical examination, chest radiographs (86-20-51 and 89-20-51) and computed tomography (CT) scans (89-20-52 and 95-20-52). All patients had ECOG performance scores ranging from 0 to 2 . Regarding the delivery of PCI, study guidelines differed slightly. In 86-20-51, ESCLC patients were to receive TRT and PCI (30Gy/15) if they had a complete response outside of the chest. All patients with LSCLC were to receive TRT and PCI (30Gy/15) if they had stable disease or a better response to the initial therapy (chemotherapy). Trial 89-20-51 and trial 89-20-52 included only patients with ESCLC or LSCLC, respectively, who were to receive PCI (30Gy/15) if a complete response after initial therapy was achieved. Trial 95-20-53 included only patients with LSCLC who were to receive PCI after any response to initial therapy. Only 95-20-53 utilized the most common current PCI regimen (25 Gy/10), which was administered after initial chemotherapy but before TRT. All trials specified brain imaging (contrast-enhanced CT or MRI) at the time of initial staging as well as prior to the delivery of PCI in order to exclude patients with metastatic disease already present in the brain. Patients had follow-up performed as dictated by the trial in which they were enrolled. Institutional Review Boards at the study sites had approved these trials and all participants provided written informed consent. See Table 1 for further information regarding the details of the four individual trials.

\section{Statistical Methods}

Baseline patient and disease characteristics between the PCI and no-PCI groups were compared utilizing the chi-square test for categorical data and the Wilcoxon rank sum test for continuous data. Survival was compared using a landmark analysis that included only patients who had stable disease (SD) or better. Overall survival (OS) from the landmark time was assessed for the PCI and no-PCI groups, where the landmark time was the point in time at which the patients could have started PCI per their particular treatment protocol. The Kaplan-Meier method was used to compare the survival distributions for the two groups. The Cox proportional hazards model was utilized for both the univariate and multivariate analyses. The multivariate models were developed by including PCI (vs. no-PCI) and all the clinically relevant factors that were collected across all trials. These included age, gender, ECOG PS, and stage. We also further adjusted for complete response to chemotherapy to take into account the association between receipt of PCI and response to chemotherapy. Score and likelihood ratio $P$ values were reported for the univariate and multivariate models, respectively, after stratifying by study, which takes into account key differences between the trials. The Wald test was used to examine the significance of parameters with more than two categories. Hazard ratios (HRs) and their associated 95\% confidence intervals (CIs) were calculated for univariate and multivariate results. Chi-square or Fisher's exact tests were used to compare the adverse event rates between PCI and no-PCI groups, focusing on the grade 3 or higher adverse events. In addition, univariate logistic regression models were also 
used to assess the relationship between PCI (vs. no-PCI) and grade 3 or worse adverse events, after stratifying by study. All tests were two-sided, with $P$ values $<0.05$ indicating statistical significance.

\section{Results}

One hundred and fifty-five patients 70 years or older with LSCLC or ESCLC were identified as the study population. Of the 155 patients, 84 had LSCLC and 71 had ESCLC. The median follow-up for surviving patients was 100 months (range 61-139 months). Table 2 lists relevant patient characteristics in patients receiving PCI versus no-PCI. For the entire patient population, 91 (59\%) patients received PCI and 64 (41\%) did not receive PCI. Of the 84 patients with LSCLC, 64 (76\%) received PCI and 20 (24\%) did not receive PCI. This was a higher proportion than was seen in the 71 patients with ESCLC, where only 27 (38\%) received PCI and $44(62 \%)$ did not receive PCI. Patients receiving PCI were more likely to have an ECOG performance status of 0 and were also less likely to have an ECOG performance status of 2 than patients who did not receive PCI $(p=0.0235)$. Patients with LSCLC were more likely to undergo PCI compared to ESCLC patients $(p<0.0001)$. In addition, we looked at the association of PCI with chemotherapy response and found that patients who received PCI were more likely to have had a complete response (CR) to chemotherapy. Specifically, $57 \%$ of the patients who received PCI had a CR vs. $23 \%$ in the no-PCI group $(p<0.0001$ ). This was driven by the extensive-stage patients, where $67 \%$ of the patients who received PCI had a CR vs. only $16 \%$ in the no-PCI group ( $<<0.0001)$. No significant difference was observed in the limited-stage patients (PCI CR rate: 53\% vs. noPCI CR rate: $40 \%$; 0.3055). As such, in the multivariate landmark analyses models for OS, we further adjusted for response to chemotherapy (CR vs. no CR). Age and gender factors did not result in any apparent differences between the groups on analysis.

Regarding adverse events (AEs) for the entire patient population, grade 3 or higher overall toxicity was significantly greater in patients who received PCI ( $71.4 \%$ vs. $47.5 \%, p=0.0031$, $p$ (stratified by study) $=0.0589$ ). For the entire patient population, non-neuro and non-heme were the specific AE categories of grade 3 or higher toxicity that were significantly greater in patients who received PCI (68.1\% vs. $47.5 \%, p=0.0116, p$ [stratified by stud] $)=0.1409$ and $70.3 \%$ vs $40.7 \%, p=0.0003, p$ [stratified by study] $=0.0192$ ), where the non-heme AE differences remained significant even after running a univariate logistic model that stratified by study $(\mathrm{p}=0.0192)$. Individual toxicities separate from the larger toxicity groupings were not significantly correlated with PCI delivery except in LSCLC patients, where alopecia was noted to be significantly correlated with PCI delivery ( $40.6 \%$ vs. $10.5 \%, p=0.0148$ ). However, this relationship was no longer significant after stratifying by study ( $p=0.6049$ ). Long-term neurocognitive and quality of life data were not collected and thus could not be analyzed.

For the entire patient population, elderly patients who received PCI had better survival than patients who did not receive PCI (median survival [MS] 12.0 months vs. 7.6 months, 3-year OS $13.2 \%$ vs. $3.1 \%, \mathrm{HR}=0.53$ [95\% CI $0.36-0.78$ ], univariate $p=0.001$, see Figure 1 ). On multivariate analysis, the only parameter that remained significant for overall survival in the entire population was stage (ESCLC vs. LSCLC, see Table 3). Interestingly, in the LSCLC 
cohort, there was no significant difference in OS between PCI and no-PCI patients (median survival 12.2 months vs. 16.0 months, 3-year OS $17.2 \%$ vs. $10.0 \%, \mathrm{HR}=0.91[95 \% \mathrm{CI}$ 0.49-1.68], $p=0.7630$, see Figure 2, Table 4). For the ESCLC cohort, PCI patients had significantly better survival than patients who did not receive PCI (median survival 10.8 months vs. 7.1 months, 3 -year OS $3.7 \%$ vs. $0 \%, \mathrm{HR}=0.46$ [95\% CI $0.27-0.77$ ], $p=0.003$, see Figure 3). When analyzed separately, the multivariate analysis for the ESCLC cohort revealed the only parameter that remained significant for OS was the delivery of PCI (HR = 0.47 [95\% CI 0.24-0.93], $p=0.03$, see Table 5).

\section{Discussion}

Our analysis demonstrates that the OS benefit of PCI in this particular cohort of trials extends to patients with ESCLC who are 70 years and older. This is in agreement with the findings of a randomized trial examining the effect of PCI in patients with ESCLC who had any response to systemic therapy.(4) Patients receiving PCI had a significantly improved OS rate at 1 -year compared to patients who did not receive PCI $(27.1 \%$ vs. $13.3 \%, p=0.003)$. The MS, after randomization, was 6.7 months in those who received PCI compared to 5.4 months in those who did not. This trial allowed enrollment of patients up to 75 years of age. The median age in the PCI arm was 62 years, with a range of 37 to 75 years, indicating that elderly patients were indeed enrolled. However, it is not clear exactly how many patients 70 years and older were enrolled, as a subset analysis of this population has not been reported. These results are similar to the present analysis which revealed patients with ESCLC had a MS of 10.8 months (with PCI) vs. 7.1 months (without PCI) $(p=0.003$ ).

A meta-analysis published by Auperin et al. in 1999 compared PCI with no-PCI in patients who had achieved a CR to initial therapy.(2) There was a statistically significant 5.4\% improvement in 3-year OS in the PCI group. The analysis did include a small number of patients with ESCLC as well as a small number of patients 65 years of age and older, but did not specifically comment on the efficacy in PCI in the cohort of patients with a combination of both of those characteristics. Subgroup analyses found no overt evidence that any particular subgroup of patients (beyond those defined according to sex and the interval between induction therapy and randomization) benefited to a greater or lesser degree from PCI. The findings from the present study do correlate well with this meta-analysis.

In contrast, a recently reported randomized phase III trial from Japan raised the specter of a potential detrimental effect on OS of PCI in ESCLC. While obviously concerning, it has been reported in abstract form only. Thus, further comments and analysis regarding elderly patients (and other patient groups) must await scrutiny of the peer-reviewed manuscript.(8)

While our particular cohort of older patients with LSCLC did not appear to benefit from PCI, the benefit of PCI in older patients with LSCLC is supported by the results of two SEER analyses.(5, 7) In the first of these analyses, a group of 7995 patients with LSCLC diagnosed between 1988 and 1997 was identified. From that group, 670 patients were noted to have had PCI as part of their initial therapeutic course. Overall and cause-specific survival at 2, 5, and 10-years was significantly improved in patients who received PCI versus those who did not receive PCI. Multiple age group cohorts were analyzed (age <60, 60-66, 67-72, 
and $\geq 73$ ), with all age groups noted to benefit from PCI. The 5-year OS for the 67-72 years cohort was $16 \%$ in the PCI group and $10 \%$ in the no-PCI group $(p=0.0005)$. The 5-year OS for the $\geq 73$ years cohort was $10 \%$ in the PCI group and 5\% in the no-PCI group $(p<0.0001)$. (5) In the second analysis of patients with LSCLC > 70 years (with a median age of 75) PCI was associated with improved survival ( $33 \%$ vs $23 \%, p=0.028)$. Furthermore, multivariate analysis demonstrated that PCI remained an independent factor associated with OS (HR, 0.72 ; 95\% CI, $0.54-0.97$ [ $p=0.032]$ ) for those $>75$ years, but not in those $>80$ years. (7) It is not clear why a benefit from PCI was not seen in our cohort of patients with LSCLC, though it is possible that the limited patient numbers reduced the power to detect statistically significant differences between cohorts. However, it appears clear from the much larger SEER studies that elderly patients with LSCLC have the same survival benefit (approximately $5 \%$ at 5 years) as do other patients with SCLC. $(5,7)$

While the data collected for these trials was prospectively gathered, this sub-analysis was retrospective in nature. Potential biases were mitigated with multivariate analyses and adjustment for other prognostic factors. Another limitation of our data is the lack of neurocognitive and quality of life outcome data. In the past, PCI often included large daily doses (3Gy or greater) and was sometimes delivered concurrently with chemotherapy. Both of these practices were fraught with an increased risk of potentially serious long-term neurologic sequelae.(9-16) Modern PCI doses have decreased significantly and the delivery of concurrent PCI and chemotherapy is not advocated.

Regarding the specifics of PCI dosing, Le Pechoux et al. reported the results of an international randomized phase III trial (PCI 99-01, EORTC 22003-08004, Radiation Therapy Oncology Group 0212, and IFCT 99-01) comparing modern standard dose PCI (25Gy in 10 daily fractions) with higher dose PCI (36Gy in 18 daily fractions or 36Gy in 24 fractions, delivering $1.5 \mathrm{~Gy}$ twice daily). No benefit was seen with the higher PCI dose regimens, with $25 \mathrm{~Gy}$ in 10 daily fractions remaining the standard PCI dose.(17) In fact, the lower dose group was associated with an increase in 2-year OS $(\mathrm{p}=0.05)$. Enrolled patients had baseline and follow-up neuropsychological testing in an effort to track neuropsychological deterioration. In that trial, patients older than 60 years were more likely to experience trial-defined chronic neurotoxicity $(\mathrm{CN})$ at 12 months after PCI than patients younger than 60 years ( $83 \%$ vs. $56 \%, p=0.009)$. It should be noted that the number of patients available for analysis at that time point was quite small. Specific data for the patients 70 years or older ( 24 patients out of the total trial population of 720 patients) were not published. Trial-defined $\mathrm{CN}$ was also significantly more frequent in patients on the higher dose PCI arms compared to the standard dose PCI arm $(89 \%$ for $1.5 \mathrm{~Gy} \times 24,85 \%$ for $1.8 \mathrm{~Gy} \times 20,60 \%$ for $2.5 \mathrm{~Gy} \times 10, p=0.02) .(18)$

Given the uncertainties and potential for neurotoxicity of PCI in the elderly patient population, future studies would ideally be performed to better delineate how it can be effectively administered with less risk. In the current research funding environment, it is highly unlikely that robust prospective data regarding the efficacy and toxicity of PCI specifically in elderly SCLC patients will ever be available. Thus, providers and patients will be left with data in the form of retrospective/subgroup analyses and meta-analyses. Potential insight, however flawed, can potentially be gained by examining data from patients with 
brain metastases treated with whole brain radiotherapy (WBRT). A recently published phase III trial (RTOG 0614) explored the utility of memantine in patients receiving WBRT for brain metastases. Patients receiving memantine had a significantly longer time to cognitive decline, with a probability of trial-defined cognitive function failure at 24 weeks of $54 \%$ vs. $65 \%$ on the placebo arm (HR $0.78, p=0.01$ ). Patients on the memantine arm had superior results in executive function at $8(p=0.008)$ and 16 weeks $(p=0.0041)$ as well as processing speed $(p=0.0137)$ and delayed recognition $(p=0.0149)$ at 24 weeks. The primary endpoint of the trial, delayed recall at 24 weeks, demonstrated a trend in favor of the memantine arm but was not statistically significant $(p=0.059)$. This was thought to be related to significant patient loss.(19) Memantine was well tolerated, with a toxicity profile similar to placebo, making it a potentially attractive agent to consider in patients undergoing PCI.

Modern radiotherapeutic techniques may also provide an avenue for enhancing the therapeutic ratio in PCI patients. Hippocampal sparing WBRT was recently demonstrated to significantly reduce the rate of cognitive decline in patients with brain metastases enrolled on the RTOG 0933 trial.(20) This is leading to a phase III trial in patients with brain metastases and has spurred interest in utilizing hippocampal sparing techniques in PCI patients.

\section{Conclusion}

The survival rates of patients with SCLC are quite modest and PCI has been proven to enhance OS. $(2,4,5,7)$ The data presented support the role of PCI in elderly patients with SCLC as it was associated with a significant survival advantage. Many oncologists hesitate to recommend PCI, especially to elderly patients, due to concerns regarding toxicity. However, failure to administer PCI appears to jeopardize the survival of all patients with SCLC who respond to initial therapy, including the elderly.

\section{Acknowledgments}

This study was conducted as a collaborative effort of the North Central Cancer Treatment Group/Alliance for Clinical Trials in Oncology Foundation and Mayo Clinic. The content is solely the responsibility of the authors and does not necessarily represent the views of the National Cancer Institute or the National Institutes of Health.

Funding

Public Health Service (CA-25224, CA-37404, CA-35267, CA-35431, CA-63848, CA-63849, CA-35113, CA-35103, CA-35415, CA-35101, CA35119, CA-35090)

\section{References}

1. Arriagada R, Le Chevalier T, Riviere A, Chomy P, Monnet I, Bardet E, et al. Patterns of failure after prophylactic cranial irradiation in small-cell lung cancer: analysis of 505 randomized patients. Ann Oncol. 2002 May; 13(5):748-54. [PubMed: 12075744]

2. Auperin A, Arriagada R, Pignon JP, Le Pechoux C, Gregor A, Stephens RJ, et al. Prophylactic cranial irradiation for patients with small-cell lung cancer in complete remission. Prophylactic Cranial Irradiation Overview Collaborative Group. The New England journal of medicine. 1999 Aug 12; 341(7):476-84. [PubMed: 10441603]

3. Schild SE, Foster NR, Meyers JP, Ross HJ, Stella PJ, Garces YI, et al. Prophylactic cranial irradiation in small-cell lung cancer: findings from a North Central Cancer Treatment Group Pooled Analysis. Ann Oncol. 2012 Nov; 23(11):2919-24. [PubMed: 22782333] 
4. Slotman B, Faivre-Finn C, Kramer G, Rankin E, Snee M, Hatton M, et al. Prophylactic cranial irradiation in extensive small-cell lung cancer. The New England journal of medicine. 2007 Aug 16; 357(7):664-72. [PubMed: 17699816]

5. Patel S, Macdonald OK, Suntharalingam M. Evaluation of the use of prophylactic cranial irradiation in small cell lung cancer. Cancer. 2009 Feb 15; 115(4):842-50. [PubMed: 19117355]

6. Jett JR, Schild SE, Kesler KA, Kalemkerian GP. Treatment of Small Cell Lung Cancer: Diagnosis and Management of Lung Cancer, 3rd ed: American College of Chest Physicians Evidence-Based Clinical Practice Guidelines. Chest. 2013 May; 143(5 Suppl):e400S-19S. [PubMed: 23649448]

7. Eaton BR, Kim S, Marcus DM, Prabhu R, Chen Z, Ramalingam SS, et al. Effect of prophylactic cranial irradiation on survival in elderly patients with limited-stage small cell lung cancer. Cancer. 2013 Nov 1; 119(21):3753-60. [PubMed: 23921891]

8. Seto T, Takahashi T, Yamanaka T, Harada H, Nokihara H, Saka H, et al. Prophylactic cranial irradiation (PCI) has a detrimental effect on the overall survival (OS) of patients (pts) with extensive disease small cell lung cancer (ED-SCLC): Results of a Japanese randomized phase III trial. J Clin Oncol. 2014; 32(5S) Abstract 7503.

9. Johnson BE, Becker B, Goff WB 2nd, Petronas N, Krehbiel MA, Makuch RW, et al. Neurologic, neuropsychologic, and computed cranial tomography scan abnormalities in 2- to 10-year survivors of small-cell lung cancer. J Clin Oncol. 1985 Dec; 3(12):1659-67. [PubMed: 2999346]

10. Johnson BE, Patronas N, Hayes W, Grayson J, Becker B, Gnepp D, et al. Neurologic, computed cranial tomographic, and magnetic resonance imaging abnormalities in patients with small-cell lung cancer: further follow-up of 6- to 13-year survivors. J Clin Oncol. 1990 Jan; 8(1):48-56. [PubMed: 2153196]

11. Catane R, Schwade JG, Yarr I, Lichter AS, Tepper JE, Dunnick NR, et al. Follow-up neurological evaluation in patients with small cell lung carcinoma treated with prophylactic cranial irradiation and chemotherapy. International journal of radiation oncology, biology, physics. $1981 \mathrm{Jan} ;$ 7(1): $105-9$.

12. Fonseca R, O’Neill BP, Foote RL, Grill JP, Sloan JA, Frytak S. Cerebral toxicity in patients treated for small cell carcinoma of the lung. Mayo Clinic proceedings. 1999 May; 74(5):461-5. [PubMed: 10319075]

13. Shaw EG, Su JQ, Eagan RT, Jett JR, Maksymiuk AW, Deigert FA. Prophylactic cranial irradiation in complete responders with small-cell lung cancer: analysis of the Mayo Clinic and North Central Cancer Treatment Group data bases. Journal of clinical oncology : official journal of the American Society of Clinical Oncology. 1994 Nov; 12(11):2327-32. [Clinical Trial Clinical Trial, Phase II Clinical Trial, Phase III Comparative Study Multicenter Study Randomized Controlled Trial]. [PubMed: 7964948]

14. Glantz MJ, Choy H, Yee L. Prophylactic cranial irradiation in small cell lung cancer: rationale, results, and recommendations. Seminars in oncology. 1997 Aug; 24(4):477-83. [Review]. [PubMed: 9280227]

15. Ball DL, Matthews JP. Prophylactic Cranial Irradiation: More Questions Than Answers. Seminars in radiation oncology. 1995 Jan; 5(1):61-8. [PubMed: 10717128]

16. Tomio L, Romano M, Zanchin G, Carollo C, Amista P, Loreggian L, et al. Ultrarapid high-dose course of prophylactic cranial irradiation in small-cell lung cancer: evaluation of late neurologic morbidity in 16 long-term survivors. American journal of clinical oncology. 1998 Feb; 21(1):8490. [PubMed: 9499267]

17. Le Pechoux C, Dunant A, Senan S, Wolfson A, Quoix E, Faivre-Finn C, et al. Standard-dose versus higher-dose prophylactic cranial irradiation (PCI) in patients with limited-stage small-cell lung cancer in complete remission after chemotherapy and thoracic radiotherapy (PCI 99-01, EORTC 22003-08004, RTOG 0212, and IFCT 99-01): a randomised clinical trial. The lancet oncology. 2009 May; 10(5):467-74. [PubMed: 19386548]

18. Wolfson AH, Bae K, Komaki R, Meyers C, Movsas B, Le Pechoux C, et al. Primary Analysis of a Phase II Randomized Trial Radiation Therapy Oncology Group (RTOG) 0212: Impact of Different Total Doses and Schedules of Prophylactic Cranial Irradiation on Chronic Neurotoxicity and Quality of Life for Patients With Limited-Disease Small-Cell Lung Cancer. International journal of radiation oncology, biology, physics. 2011 Sep 1; 81(1):77-84. 
19. Brown PD, Pugh S, Laack NN, Wefel JS, Khuntia D, Meyers C, et al. Memantine for the prevention of cognitive dysfunction in patients receiving whole-brain radiotherapy: a randomized, double-blind, placebo-controlled trial. Neuro Oncol. 2013 Aug 16.

20. Gondi V, Mehta MP, Pugh S, Tome WA, Kanner A, Caine C, et al. Memory Preservation with Conformal Avoidance of the Hippocampus during Whole-Brain Radiotherapy (WBRT) for Patients with Brain Metastases: Primary Endpoint Results of RTOG 0933. International journal of radiation oncology, biology, physics. 2013; 87(2S) 


\section{Synopsis/Summary}

This pooled analysis examines the role of prophylactic cranial irradiation in the elderly patient population. 


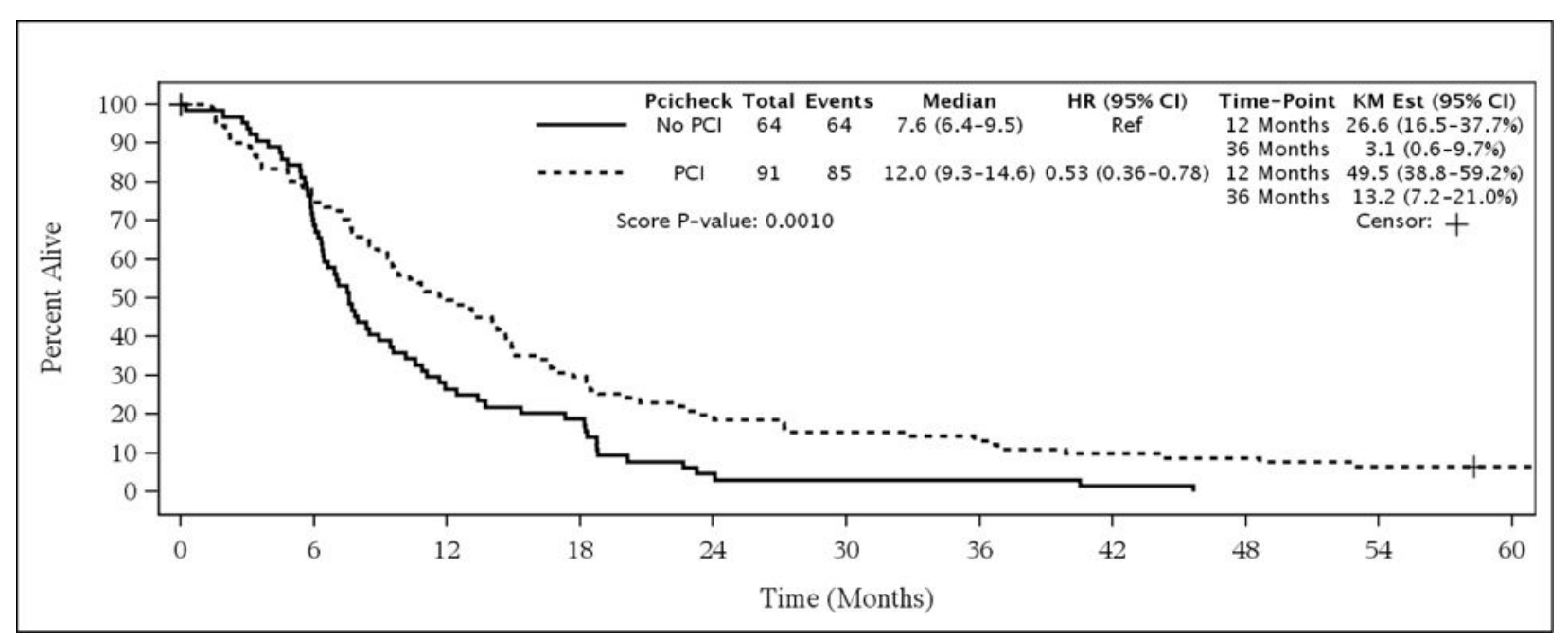

Figure 1.

Survival in All Patients 


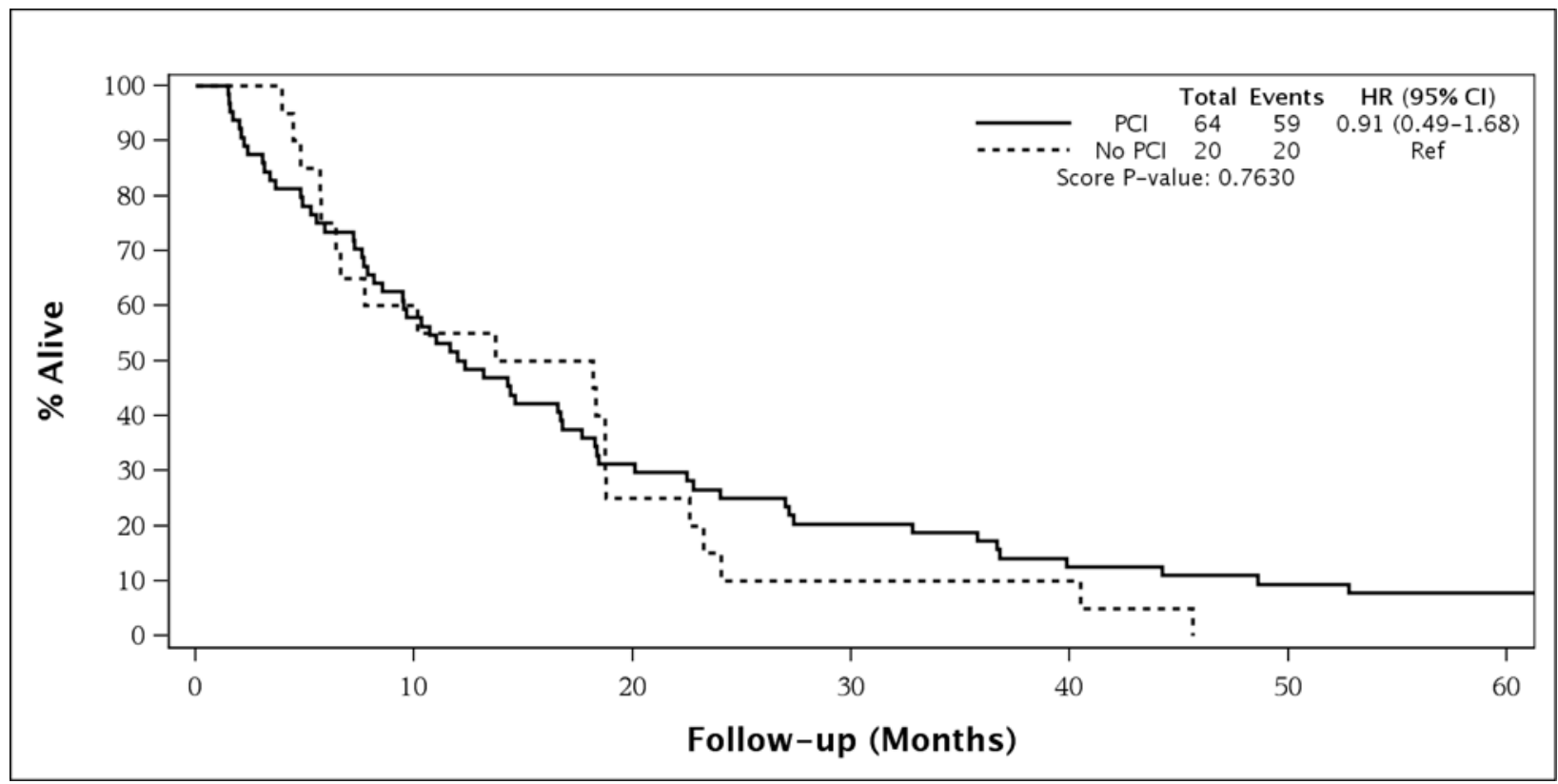

Figure 2.

Survival in Patients with Limited Stage Small Cell Lung Cancer 


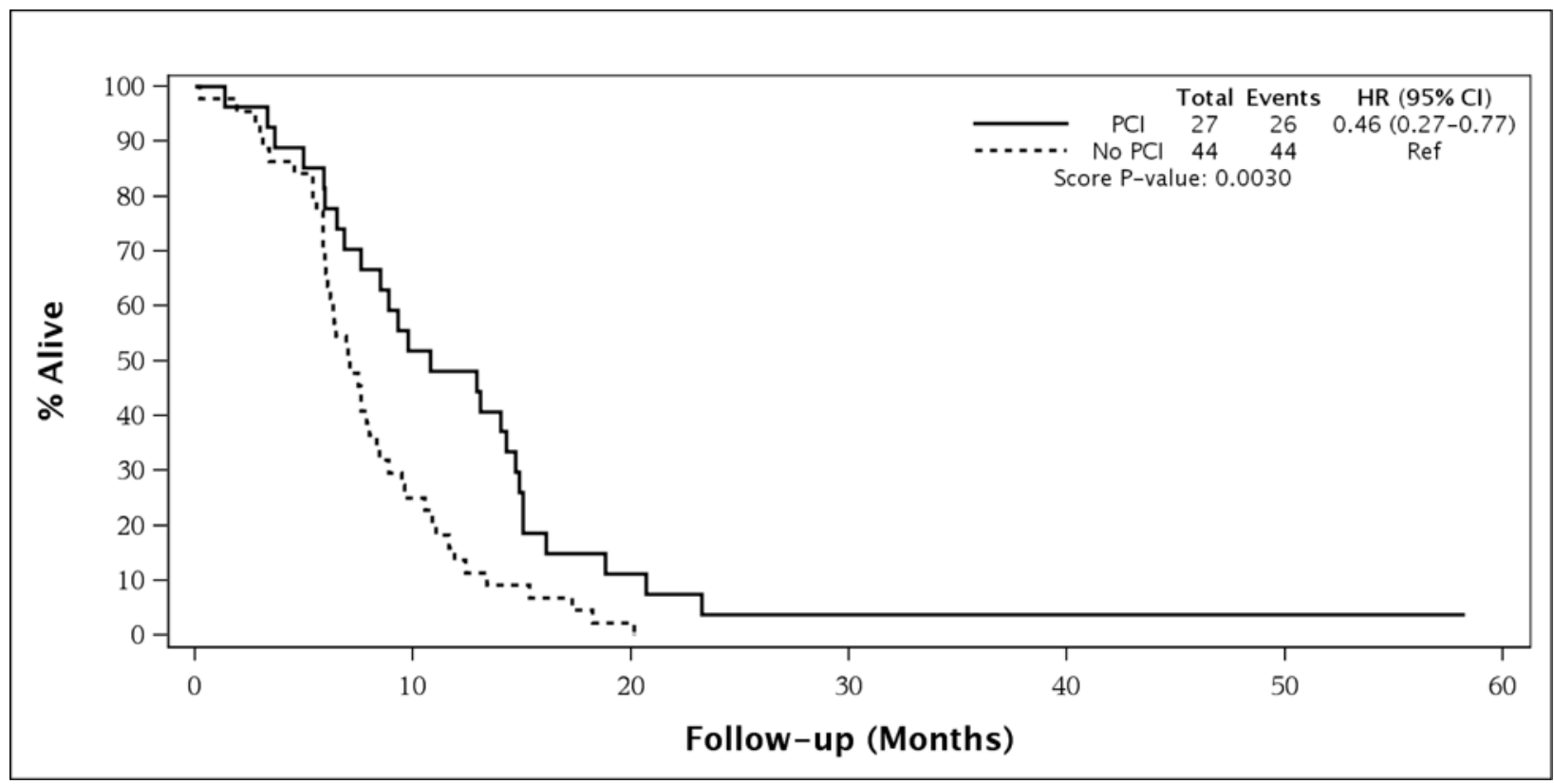

Figure 3.

Survival in Patients with Extensive Stage Small Cell Lung Cancer 
Table 1

Study Characteristics

\begin{tabular}{|c|c|c|c|c|}
\hline Protocol & $86-20-51$ & $89-20-51$ & $89-20-52$ & $95-20-53$ \\
\hline $\begin{array}{l}\mathrm{N} \text { (stable or better included in } \\
\text { landmark analysis) }\end{array}$ & 83 & 33 & 30 & 9 \\
\hline Stage & $\begin{array}{l}\text { LSCLC: } 45(54.2 \%) \\
\text { ESCLC: } 38(45.8 \%)\end{array}$ & ESCLC & LSCLC & LSCLC \\
\hline \multirow[t]{4}{*}{ Best Response } & CR $35(42.2 \%)$ & CR $12(36.4 \%)$ & CR $21(70.0 \%)$ & CR $1(11.1 \%)$ \\
\hline & PR 17 (20.5\%) & PR $7(21.2 \%)$ & PR $4(13.3 \%)$ & PR 4 (44.4\%) \\
\hline & REGR 27 (32.5\%) & REGR $12(36.4 \%)$ & REGR $4(13.3 \%)$ & REGR $3(33.3 \%)$ \\
\hline & SD $4(4.8 \%)$ & SD $2(6.1 \%)$ & SD $1(3.3 \%)$ & SD $1(11.1 \%)$ \\
\hline $\begin{array}{l}\text { Median subsequent survival } \\
\text { (95\% confidence interval) }\end{array}$ & $9.6(7.7-12.0)$ & $8.0(6.9-11.7)$ & $10.2(6.6-18.4)$ & $18.2(1.5-27.2)$ \\
\hline $\begin{array}{l}\text { Frequency }(\%) \text { that received } \\
\text { PCI }\end{array}$ & $59(71.1 \%)$ & $9(27.3 \%)$ & $15(50.0 \%)$ & $8(88.9 \%)$ \\
\hline PCI Dose & $30 \mathrm{~Gy} / 15$ & $30 \mathrm{~Gy} / 15$ & $30 \mathrm{~Gy} / 15$ & $25 \mathrm{~Gy} / 10$ \\
\hline $\begin{array}{l}\text { Frequency }(\%) \text { that received } \\
\text { TRT }\end{array}$ & $62(74.7 \%)$ & $13(39.4 \%)$ & $25(83.3 \%)$ & $2(22.2 \%)$ \\
\hline Date Opened & March 1987 & August 1990 & September 1990 & October 1996 \\
\hline Date Closed & August 1990 & July 1993 & November 1996 & March 1999 \\
\hline Phase & III & III & III & II \\
\hline Performance Status (PS) & $0-2$ & $0-2$ & $0-2$ & $0-1$ \\
\hline Agents $^{a}$ & $\begin{array}{l}\text { A:B:C:D: etoposide + } \\
\text { cisplatin + CAV + TRT + } \\
\text { PCI (if CR outside of chest } \\
\text { for ESCLC; if stable or } \\
\text { better response for } \\
\text { LSCLC) + recombinant } \\
\text { gamma interferon or } \\
\text { observation (for CR pts } \\
\text { only) }\end{array}$ & $\begin{array}{l}\text { A: etoposide + cisplatin } \\
+ \text { Megace + TRT + PCI } \\
\text { (for CR pts only) } \\
\text { B: etoposide + cisplatin } \\
\text { + placebo + TRT + PCI } \\
\text { (for CR pts only) }\end{array}$ & $\begin{array}{l}\text { A: etoposide + cisplatin } \\
+ \text { standard TRT }+ \\
\text { etoposide + cisplatin + } \\
\text { PCI (for CR pts only) } \\
\text { B: etoposide + cisplatin } \\
+ \text { AHSCTRT + } \\
\text { etoposide + cisplatin + } \\
\text { PCI (for CR pts only) }\end{array}$ & $\begin{array}{l}\text { A: etoposide + } \\
\text { cisplatin + PCI (if } \\
\text { regression or better on } \\
\text { chest X-ray) + } \\
\text { AHSCTRT + } \\
\text { etoposide + cisplatin }\end{array}$ \\
\hline
\end{tabular}

\footnotetext{
${ }^{a}$ Patients with central nervous system only progression, typically allowed whole brain radiation therapy across all studies.

CAV = cyclophosphamide, doxorubicin, vincristine; AHSCTRT, accelerated hyperfractionated split-course thoracic radiotherapy; CR, complete response; ESCLC, extensive small-cell lung cancer; LSCLC, limited small-cell lung cancer; Megace, megestrol acetate; mos, months; PCI, prophylactic cranial irradiation; PR, partial response; pts, patients; SD, stable disease; TRT, thoracic radiation therapy.
} 
Table 2

Comparison of PCI (prophylactic cranial irradiation) versus No PCI Across All Patients

\begin{tabular}{|c|c|c|c|c|}
\hline & No PCI $(N=64)$ & PCI $(N=91)$ & Total $(\mathrm{N}=155)$ & $P$ value $c$ \\
\hline Age & & & & $0.2908^{a}$ \\
\hline Mean (standard deviation) & $73.1(2.6)$ & $72.6(2.3)$ & $72.8(2.4)$ & \\
\hline Median & 73.0 & 72.0 & 72.0 & \\
\hline Range & $(70.0-80.0)$ & $(70.0-79.0)$ & $(70.0-80.0)$ & \\
\hline Sex & & & & $0.5038^{b}$ \\
\hline Female & $24(37.5 \%)$ & $39(42.9 \%)$ & $63(40.6 \%)$ & \\
\hline Male & $40(62.5 \%)$ & $52(57.1 \%)$ & $92(59.4 \%)$ & \\
\hline Performance Status & & & & $\mathbf{0 . 0 2 3 5}^{b}$ \\
\hline 0 & $15(23.4 \%)$ & $34(37.4 \%)$ & $49(31.6 \%)$ & \\
\hline 1 & $31(48.4 \%)$ & $46(50.5 \%)$ & $77(49.7 \%)$ & \\
\hline 2 & $18(28.1 \%)$ & $11(12.1 \%)$ & $29(18.7 \%)$ & \\
\hline Extent of Disease & & & & $<0.0001 b$ \\
\hline Limited & $20(31.3 \%)$ & $64(70.3 \%)$ & $84(54.2 \%)$ & \\
\hline Extensive ( 0 or 1 met site) & $23(35.9 \%)$ & $18(19.8 \%)$ & $41(26.5 \%)$ & \\
\hline Extensive $(2+$ mets sites $)$ & $21(32.8 \%)$ & $9(9.9 \%)$ & $30(19.4 \%)$ & \\
\hline \multicolumn{5}{|l|}{ Wilcoxon rank sum test } \\
\hline$b_{\text {Chi-square test }}$ & & & & \\
\hline
\end{tabular}




\section{Table 3}

Multivariate Analysis of Survival (N = 155) Parameter

\begin{tabular}{llr}
\hline & Hazard Ratio (95\% Confidence Interval) & P value $^{\boldsymbol{a}, \boldsymbol{c}}$ \\
\hline PCI (versus no PCI) & $0.743(0.48-1.15)$ & 0.1872 \\
Age (1-year increase) & $1.016(0.95-1.09)$ & 0.6745 \\
Male (versus female) & $1.080(0.75-1.56)$ & 0.6802 \\
Performance Score & & 0.5808 \\
$\quad$ PS 1 (versus PS 0) & $0.844(0.58-1.23)$ & $0.3817^{b}$ \\
PS 2 (versus PS 0) & $1.014(0.59-1.74)$ & $0.9606^{b}$ \\
Stage & & $\mathbf{0 . 0 0 7 2}$ \\
ESCLC (0 or 1 metastatic site) versus LSCLC & $2.141(1.25-3.68)$ & $\mathbf{0 . 0 0 5 9}$ \\
ESCLC (>1 metastatic site) versus LSCLC & $2.608(1.38-4.93)$ & $\mathbf{0 . 0 0 3 1}$ \\
Response: CR (versus no CR) & $0.716(0.49-1.05)$ & 0.0836 \\
\hline
\end{tabular}

a Likelihood ratio P value from a Cox model, stratified by study.

$b_{\text {Wald P values. }}$

$c_{\text {Significant values are in bold }}$

CR, complete response; ESCLC, extensive small-cell lung cancer; LSCLC, limited small-cell lung cancer; PCI, prophylactic cranial irradiation. 
Table 4

Multivariate Analysis of Survival in Limited Stage Patients $(\mathrm{N}=84)$

\begin{tabular}{llc}
\hline Parameter & Hazard Ratio (95\% Confidence Interval) & P value $^{\boldsymbol{a}, \boldsymbol{c}}$ \\
\hline PCI (versus no PCI) & $1.136(0.58-2.21)$ & 0.7055 \\
Age (1-year increase) & $1.095(0.99-1.21)$ & 0.0753 \\
Male (versus female) & $0.898(0.54-1.49)$ & 0.6786 \\
Performance Score & & 0.3845 \\
PS 1 (versus PS 0) & $0.833(0.52-1.34)$ & $0.4479 b$ \\
PS 2 (versus PS 0) & $1.575(0.62-4.03)$ & $0.3432^{b}$ \\
CR (versus no CR) & $0.829(0.51-1.35)$ & 0.4539 \\
\hline
\end{tabular}

a Likelihood ratio $\mathrm{P}$ value from a Cox model, stratified by study.

$b_{\text {Wald } \mathrm{P} \text { values. }}$

$c_{\text {Significant values are in bold }}$

CR, complete response; ESCLC, extensive small-cell lung cancer; LSCLC, limited small-cell lung cancer; PCI, prophylactic cranial irradiation. 
Table 5

Multivariate Analysis of Survival in Extensive Stage Patients ( $\mathrm{N}=71)$

\begin{tabular}{llr}
\hline Parameter & Hazard Ratio (95\% Confidence Interval) & P value $^{\boldsymbol{a}, \boldsymbol{c}}$ \\
\hline PCI (versus no PCI) & $0.471(0.24-0.93)$ & $\mathbf{0 . 0 3 0 0}$ \\
Age (1-year increase) & $0.912(0.82-1.02)$ & 0.1007 \\
Male (versus female) & $1.478(0.84-2.61)$ & 0.1752 \\
Performance Score & & 0.8472 \\
$\quad$ PS 1 (versus PS 0) & $0.909(0.46-1.79)$ & $0.7837^{b}$ \\
PS 2 (versus PS 0) & $1.078(0.49-2.38)$ & $0.8525^{b}$ \\
Stage & & 0.3757 \\
$\quad>1$ metastatic site) versus (0 or 1 metastatic site) & $1.268(0.75-2.14)$ & $0.3744^{b}$ \\
Response: CR (versus no CR) & $0.698(0.34-1.44)$ & 0.3241 \\
\hline
\end{tabular}

a Likelihood ratio P value from a Cox model, stratified by study.

$b$ Wald P values.

$c_{\text {Significant values are in bold }}$

CR, complete response; ESCLC, extensive small-cell lung cancer; LSCLC, limited small-cell lung cancer; PCI, prophylactic cranial irradiation. 